\title{
Pseudoxanthoma elasticum presenting as akinetic-rigid parkinsonism and dementia
}

\section{Pseudoxantoma elástico se apresentando como parkinsonismo acinético-rígido e demência}

Paulo Victor Sgobbi de SOUZA', Igor Braga FARIAS', Bruno de Mattos Lombardi BADIA1', Luiz Henrique Libardi SILVA', Daniel Delgado SENEOR1, Vitor Dias Gomes Barrios MARIN, Carlos Alberto Castro TEIXEIRA JÚNIOR', Marco Antônio Troccoli CHIEIA', Wladimir Bocca Vieira de Rezende PINTO', Acary Souza Bulle OLIVEIRA'

A 54-year-old man presented with a three-year history of progressive cognitive decline, abulia, apathy, slowing movements and global rigidity. Medical history, confirmed by skin biopsy, revealed a definite diagnosis of pseudoxanthoma elasticum. Examination disclosed angioid streaks at funduscopic evaluation, skin lesions (Figure 1), bradykinesia and marked symmetrical akinetic-rigid parkinsonism with cogwheel rigidity. Neuroimaging studies showed multiple lacunar infarcts in the basal ganglia and brainstem. Tc-99m SPECT imaging showed bilateral frontal hypoperfusion (Figure 2).

Pseudoxanthoma elasticum is a rare autosomal recessive ectopic mineralization disorder ${ }^{1,2}$, which can present a complex neurological picture due to severe vascular compromise, rarely with movement disorders ${ }^{1,2}$.
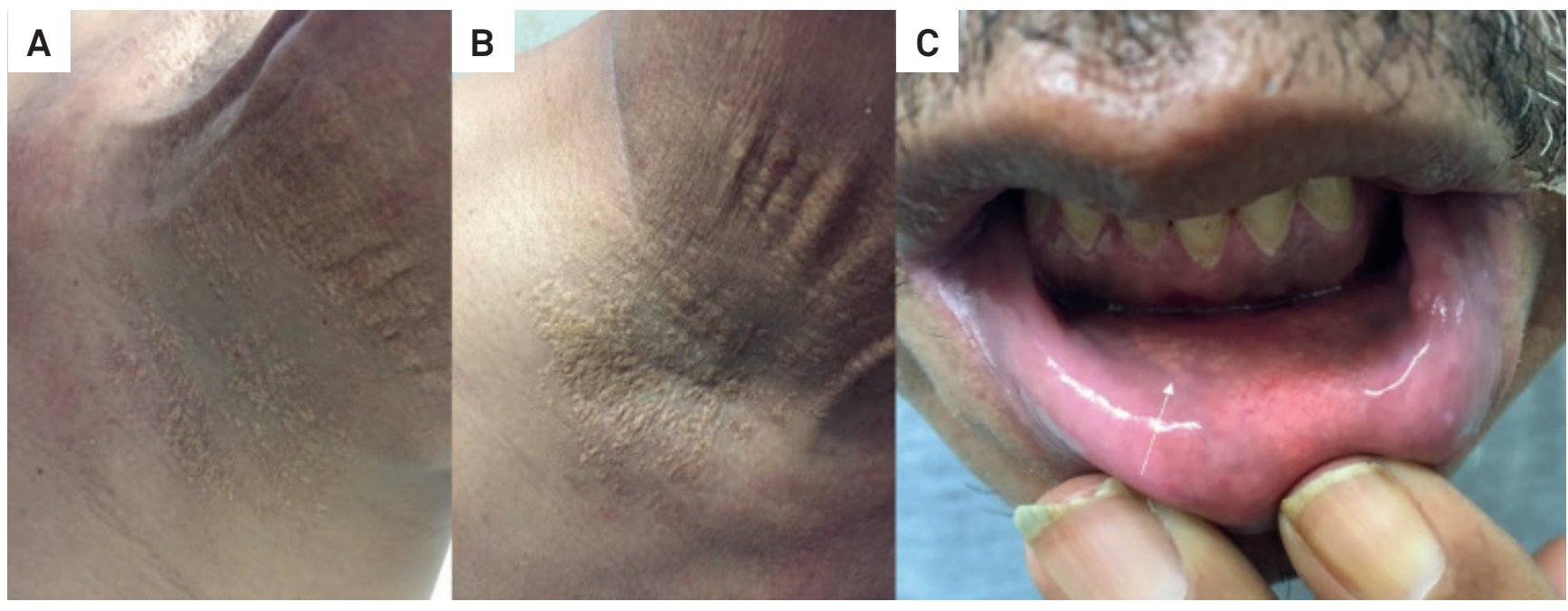

Figure 1. Typical skin lesions in pseudoxanthoma elasticum. Note the presence of (A,B) axillary and neck yellowish papules and plaques with cobblestone appearance and (C) mucosal lesions in the inner aspect of the lower lip (white arrow).

\footnotetext{
Universidade Federal de São Paulo, Departamento de Neurologia e Neurocirurgia, São Paulo SP, Brasil.

Paulo Victor Sgobbi de Souza (iD https://orcid.org/0000-0002-7416-7108; Bruno de Mattos Lombardi Badia iD https://orcid.org/0000-0001-7360-0494; Wladimir Bocca Vieira de Rezende Pinto (iD) https://orcid.org/0000-0002-0150-525X; Acary Souza Bulle Oliveira (iD https://orcid.org/0000-0002-6986-4937 Correspondence: Wladimir Bocca Vieira de Rezende Pinto; Departamento de Neurologia e Neurocirurgia da UNIFESP; Rua Estado de Israel, 899; $04022-002$ São Paulo SP, Brasil; E-mail:wladimirbvrpinto@gmail.com 


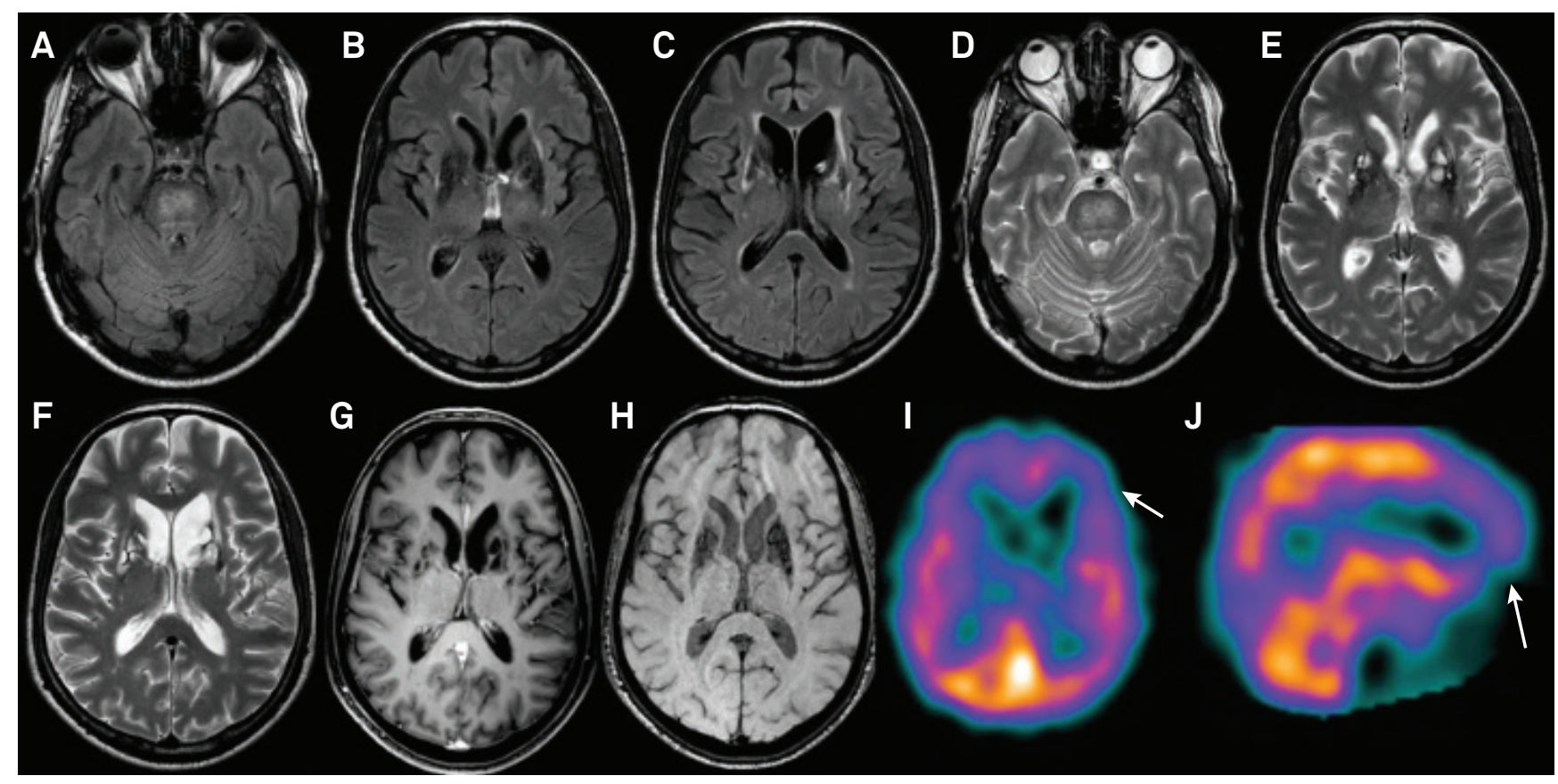

Figure 2. Neuroimaging findings in pseudoxanthoma elasticum. Axial brain MR imaging showing multiple pontine and bilateral basal ganglia lacunar infarcts with iron deposition in FLAIR sequence (A-C), T2-weighted imaging (D-F), T1-weighted imaging (G) and SWI sequences (H). (I,J) Brain Tc-99m SPECT disclosing bilateral frontal hypoperfusion (white arrows).

\section{References}

1. Chassaing N, Martin L, Calvas P, Le Bert M, Hovnanian A. Pseudoxanthoma elasticum: a clinical, pathophysiological and genetic update including 11 novel ABCC6 mutations. J Med Genet 2005 Dec;42(12):881-92. https://doi.org/10.1136/jmg.2004.030171
2. Renard D, Castelnovo G, Jeanjean L, Perrochia H, Brunel H, Labauge P. Teaching neuroimage: microangiopathic complications in pseudoxanthoma elasticum. Neurology. 2008 Nov;71(22):e69. https://doi.org/10.1212/01.wnl.0000335934.11120.83 